
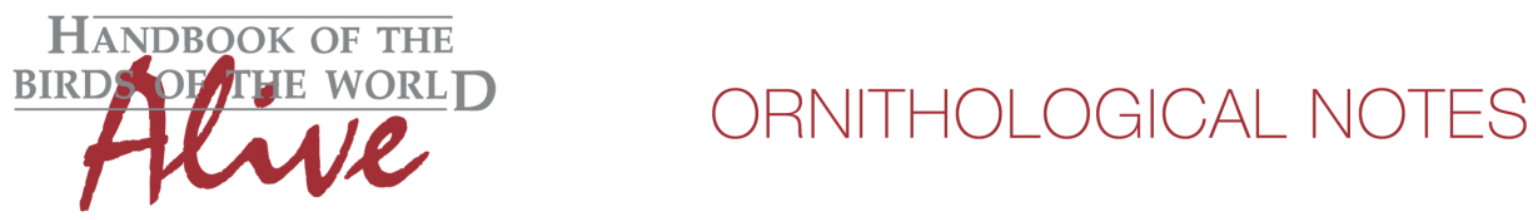

\title{
Notes on the vocalizations of Olive-brown Oriole (Oriolus melanotis)
}

\author{
Peter Boesman
}

In the following we briefly analyze and compare voice of the two races of Olive-brown Oriole (Oriolus melanotis). We also try to quantify the extent of any vocal differences using the criteria proposed by Tobias et al. (2010), as a support for taxonomic review.

We have made use of sound recordings available on-line from Xeno Canto (XC) and Macaulay Library (ML).

We have located recordings of race finschi from Wetar, and of race melanotis from both Timor and Roti. We have measured a number of basic sound parameters and calculated effect sizes:

finschi $(n=10)$

Song often consists of a single loud whistle, which often rises in pitch before dropping.

Sometimes whistle is preceded by one or (rarely) a few very short notes (Fig. 1).

$\begin{array}{llll} & \text { range } & \text { Average } & \text { Standard Dev. } \\ \text { min.freq. } & 580-1600 \mathrm{~Hz} & 889 \mathrm{~Hz} & 298 \mathrm{~Hz} \\ \text { max. freq. } & 2300-3150 \mathrm{~Hz} & 2821 \mathrm{~Hz} & 277 \mathrm{~Hz} \\ \text { freq. range } & 1430-2500 \mathrm{~Hz} & 1930 \mathrm{~Hz} & 340 \mathrm{~Hz} \\ \text { min. phrase length } & 0.32-0.75 \mathrm{~s} & 0.50 \mathrm{~s} & 0.14 \mathrm{~s} \\ \text { max. phrase length } & 0.35-0.86 \mathrm{~s} & 0.61 \mathrm{~s} & 0.25 \mathrm{~s} \\ \text { shortest note length } & 0.025-0.46 \mathrm{~s} & 0.24 \mathrm{~s} & 0.19 \mathrm{~s} \\ \text { longest note length } & 0.35-0.50 \mathrm{~s} & 0.44 \mathrm{~s} & 0.07 \mathrm{~s} \\ \text { \# notes/phrase } & 1-3 & 1.7 & 0.85\end{array}$

melanotis $(n=10)$

Song typically consists of one or a few short notes followed by a slightly longer loud whistle, which typically drops abruptly in pitch about halfway (thus sounding bisyllabic) (Fig. 1).

$\min$. freq.

max. freq.

freq. range

min. phrase length

max. phrase length

shortest note length

longest note length

\# notes/phrase

range
$520-960 \mathrm{~Hz}$
$1520-2500 \mathrm{~Hz}$
$850-1870 \mathrm{~Hz}$
$0.52-0.98 \mathrm{~s}$
$0.55-1.00 \mathrm{~s}$
$0.03-0.18 \mathrm{~s}$
$0.24-0.54 \mathrm{~s}$
$2-5$

Average

$717 \mathrm{~Hz}$

$1906 \mathrm{~Hz}$

$1190 \mathrm{~Hz}$

$0.77 \mathrm{~s}$

$0.80 \mathrm{~s}$

$0.10 \mathrm{~s}$

$0.40 \mathrm{~s}$

3.0
Standard Dev.

$139 \mathrm{~Hz}$

$308 \mathrm{~Hz}$

$289 \mathrm{~Hz}$

$0.14 \mathrm{~s}$

$0.15 \mathrm{~s}$

$0.04 \mathrm{~s}$

$0.09 \mathrm{~s}$

0.9

Main differences in basic parameters are:

max. freq.

freq. range

effect size

min. phrase length

shortest note length

\#notes/phrase
3.12

2.35

1.93

1.02

1.49 score 


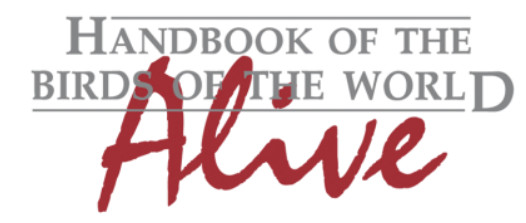

\section{ORNITHOLOGICAL NOTES}
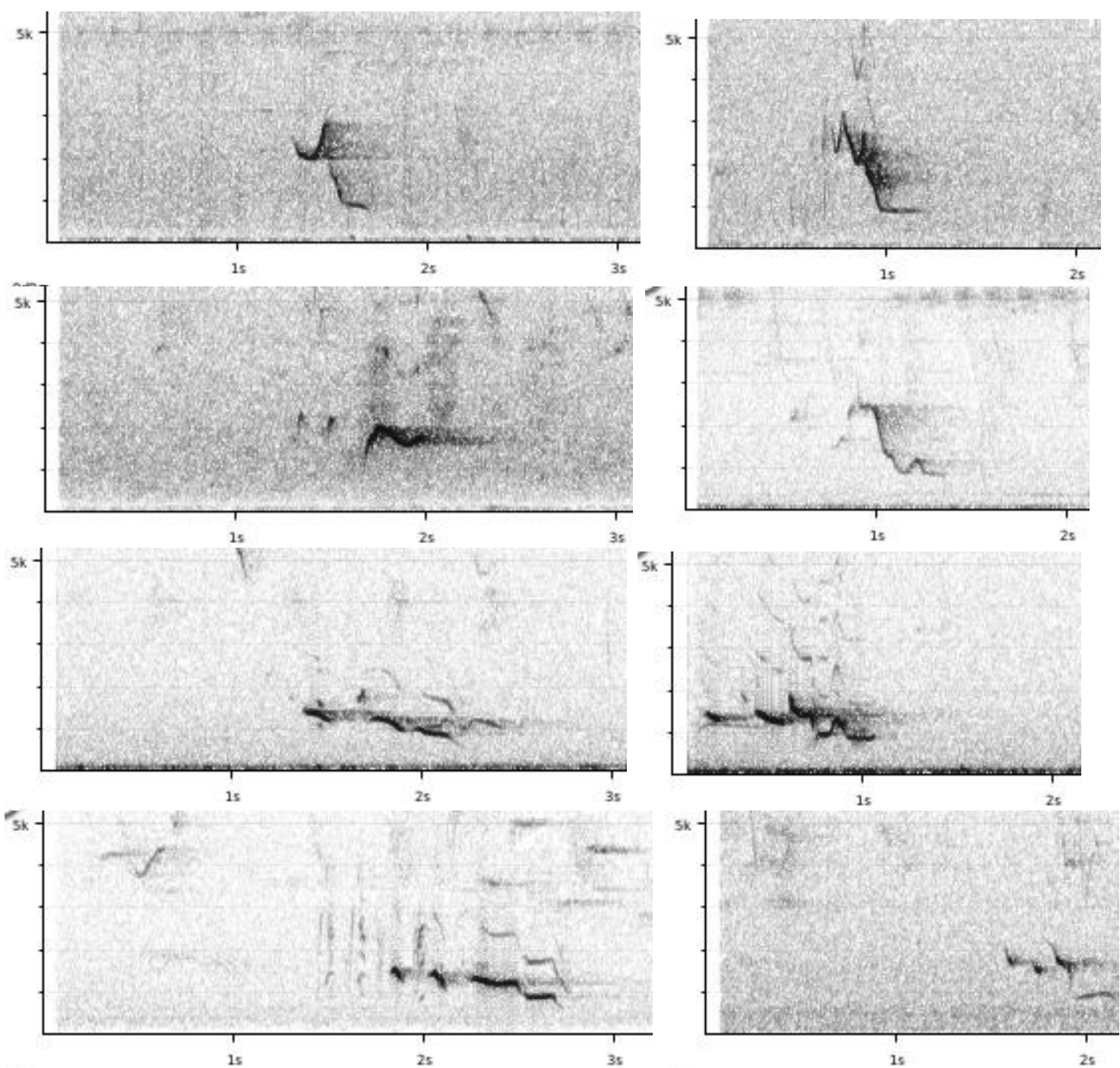

Figure 1: examples of song of race finschi (top four sonograms) and race melanotis (bottom four sonograms)

Unlike finschi, song phrase of melanotis apparently never consists of a single long whistle, whistles don't reach as high frequencies as finschi and lack the rather typical (irregular) overslurred shape of finschi, resulting in a smaller frequency range.

When applying Tobias criteria, this would lead to a total score of 3-4 (depending whether we consider max. freq. and freq. range independent or not).

This note was finalized on 9th October 2015, using sound recordings available on-line at that moment. We would like to thank in particular the sound recordists who placed their recordings for this species on XC and ML: Timothy Burr, Stijn Coleman, Hervé Jacob, Frank Lambert, Mike Nelson, Colin Trainor and Philippe Verbelen. 


\section{References}

Tobias, J.A., Seddon, N., Spottiswoode, C.N., Pilgrim, J.D., Fishpool, L.D.C. \& Collar, N.J. (2010). Quantitative criteria for species delimitation. Ibis 152(4): 724-746.

\section{Recommended citation}

Boesman, P. (2016). Notes on the vocalizations of Olive-brown Oriole (Oriolus melanotis). HBW Alive Ornithological Note 160. In: Handbook of the Birds of the World Alive. Lynx

Edicions, Barcelona. (retrieved from http://www.hbw.com/node/932090 on 18 August 2016). 